\title{
Is the APEX Score the Pinnacle of Predictive Scoring Systems of Disease Flare in Crohn's Patients with Documented Mucosal Healing?
}

\author{
Georgios I. Tsiaoussis ${ }^{1} \cdot$ Eirini Christaki ${ }^{2}$
}

Accepted: 29 June 2021 / Published online: 21 July 2021

(C) The Author(s), under exclusive licence to Springer Science+Business Media, LLC, part of Springer Nature 2021

Crohn's disease (CD) is a chronic relapsing immune-mediated disease affecting the gastrointestinal tract. Effective disease management is dependent on regularly assessing patients with $\mathrm{CD}$ for disease recurrence, which is defined according to clinical, radiographic, serologic, and endoscopic criteria. Since approximately $20 \%$ of patients with $\mathrm{CD}$ have disease limited to the small intestine and since the entire length of the small intestine cannot be visualized endoscopically, these investigations are often inconclusive. In these instances, a small bowel capsule endoscopy (SBCE) is needed to establish the diagnosis or to diagnoses a recurrence of small bowel CD [1].

The Selecting Therapeutic Targets in Inflammatory Bowel Disease (STRIDE) initiative group published clinical guidelines that defined therapeutic targets in patients with IBD [2]. The primary treatment endpoints currently include clinical and biochemical remission as well as endoscopic and histologic mucosal healing (MH) [2]. Achieving therapeutic targets provides a longer relapse-free time, fewer hospitalizations, and improved life quality. Given that CD activity and MH do not always correlate with clinical and biochemical remission, evaluation of the mucosal response to therapy might be a new indication for SBCE for patients with small bowel CD [3].

Previous studies have demonstrated that SBCE has an enhanced diagnostic yield compared with small bowel radiological imaging, ileocolonoscopy, computed tomography enterography (CTE), or magnetic resonance enterography (MRE) [4]. A diagnostic yield of $85.7 \%$ was documented in patients with established $\mathrm{CD}$, which informed treatment adjustments in $64 \%$ of those patients [5]. The Canadian

Georgios I. Tsiaoussis

tsiaoussisgeorgios@yahoo.com

1 Department of Gastroenterology, Nicosia General Hospital, CP 2103 Nicosia, Cyprus

2 Department of Internal Medicine, Medical School, University of Cyprus, Nicosia, Cyprus
Association of Gastroenterology Guidelines has also published recommendations for the use of SBCE in patients with $\mathrm{CD}$ with mucosal disease missed by ileocolonoscopy or radiographic studies and for assessing $\mathrm{MH}$ in small bowel segments inaccessible to ileocolonoscopy [6].

Proximal intestinal involvement in CD may be complicated by stenotic lesions [7]. Since jejunal involvement is associated with an increased hospitalization rate and need for surgery, it should be treated as a distinct disease phenotype due to its negative prognostic implications, similar to ileal CD that manifests as stenosis. SBCE may help identify patients who are likely to have a disease flare, given that the presence of jejunal lesions is associated with an increased risk of recurrence. Patients with proximal disease may need to be treated earlier with a more rapid step-up or a top-down approach based on SBCE findings [7, 8].

$\mathrm{MH}$ and deep remission (DR) are associated with superior outcomes in patients with $\mathrm{CD}$. A prospective cohort study in patients with $\mathrm{CD}$ reported that SBCE predicts shortterm and long-term disease relapses, and a low Lewis score (LS) might be an indicator of $\mathrm{MH}$ with clinical impact [9]. Although CD prognosis is multifactorial and affected by an interplay of factors, the contribution of SBCE to disease prognosis has not been fully evaluated, especially since most of the current data pertain to colonic MH and DR. Furthermore, the evidence regarding the optimal strategies for using SBCE in established small-bowel CD is scarce. Currently, no studies have evaluated the occurrence of disease flare in patients with small-bowel $\mathrm{CD}$ and $\mathrm{MH}$ and to determine the predictive value of SBCE use. In this issue of Digestive Diseases and Sciences, Silva et al utilized SBCE to assess small bowel $\mathrm{MH}$, evaluating only $\mathrm{CD}$ patients in clinical remission with confirmed $\mathrm{MH}$, showing for the first time how SBCE-assessed MH affects the prognosis of patients with CD [10]. This study aimed to construct a prognostic model that could act as a decision-making tool by identifying patients with small bowel $\mathrm{MH}$ who had a higher risk of relapse in the year following SBCE. The eventual goal 
Fig. 1 APEX score model for the relapse risk assessment in CD patients with MH. The medical treatment approach could be adjusted accordingly to SBCE findings and APEX scoring system in the selected group of high-risk CD patients, specifically if EIMs are present. CD, Crohn disease; $\mathrm{MH}$, mucosal healing; EIMs, extra-intestinal manifestations; PLTs, platelets; FU, follow-up; SBCE, small bowel capsule endoscopy

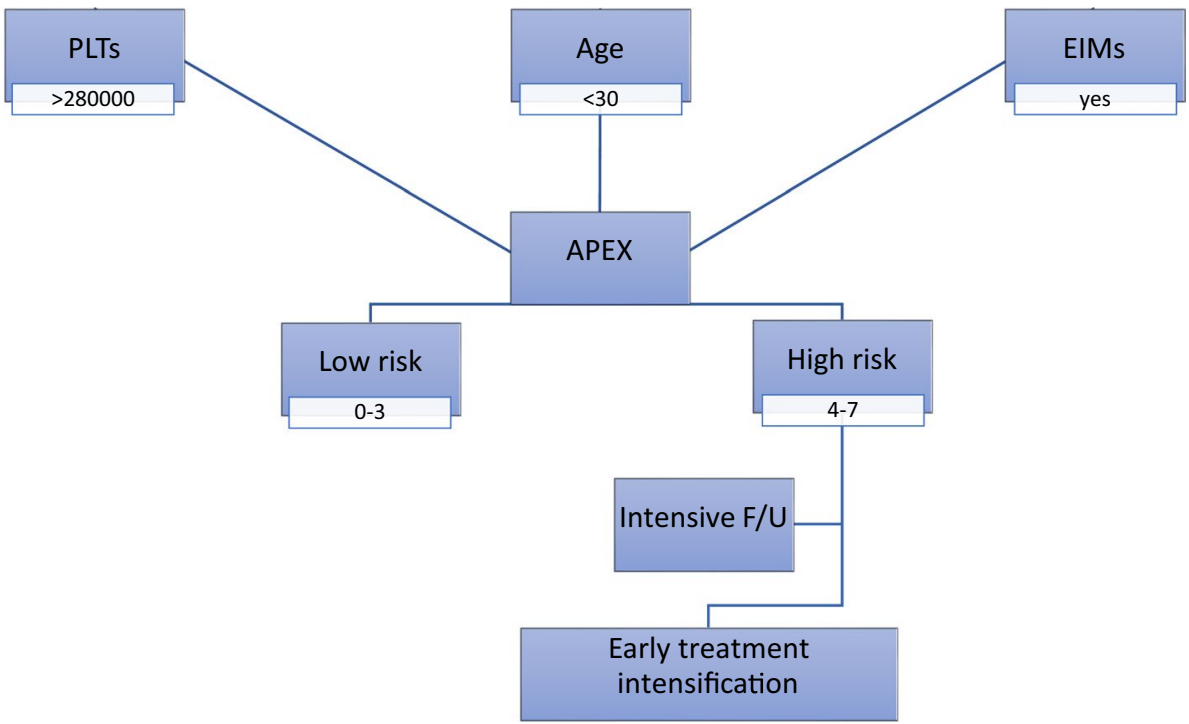

of this study was to guide further therapeutic options and follow-up therapeutic strategies (Fig. 1).

The authors reported that nearly three-quarters of their cohort remained relapse-free during the year after surveillance. Among patients who experienced disease flare, 25.5\% needed treatment plan modification, therapeutic intensification, or even hospitalization. Of those who relapsed, factors such as younger age, extra-intestinal manifestations (EIMs), and higher platelet counts may be helpful for clinicians when identifying higher-risk patients who may need earlier clinical, analytical, and endoscopic surveillance. The APEX scoring system found that age under 30 years old (A), a platelet count $>280 \times 10^{3} \mathrm{U} / \mathrm{L}(\mathrm{P})$, and the presence of EIMs (EX) were independent predictive factors of disease exacerbation. Patients in the high-risk group (4-7 points) could be considered for earlier treatment intensification and earlier follow-up intervals, as the relapse rate in this setting is around $80 \%$.

In patients with established $\mathrm{CD}$, evaluation of the extent and severity of small intestinal disease by SBCE impacts treatment decisions. SBCE can be a valuable tool for the detection and scoring of disease activity, evaluating treatment efficacy, and informing the treatment plan to either a step-up or top-down approach. It is hoped that the use of SBCE will become more widespread in individuals with CD, helping clinicians better diagnose and manage this troublesome and morbid disease.

\section{Declarations}

Conflict of interest The authors declare that they have no conflicts of interest.

\section{References}

1. Goran L, Negreanu AM, Stemate A, Negreanu L. Capsule endoscopy: Current status and role in Crohn's disease. World J Gastrointest Endosc. 2018;10:184-192. https://doi.org/10.4253/ wjge.v10.i9.184.

2. Peyrin-Biroulet L, Sandborn W, Sands BE et al. Selecting therapeutic targets in inflammatory bowel disease (STRIDE): Determining therapeutic goals for treat-to-target. Am J Gastroenterol. 2015;110:1324-38. https://doi.org/10.1038/ajg.2015.233.

3. Kopylov U, Nemeth A, Koulaouzidis A et al. Small bowel capsule endoscopy in the management of established Crohn's disease: clinical impact, safety, and correlation with inflammatory biomarkers. Inflamm Bowel Dis. 2015;21:93-100. https://doi. org/10.1097/MIB.0000000000000255.

4. Dionisio PM, Gurudu SR, Leighton JA, et al. Capsule endoscopy has a significantly higher diagnostic yield in patients with suspected and established small-bowel Crohn's disease: A metaanalysis. Am J Gastroenterol. 2010;105:1240-8 (quiz 1249). https://doi.org/10.1038/ajg.2009.713.

5. Lorenzo-Zúñiga V, de Vega VM, Domènech E, Cabré E, Mañosa M, Boix J. Impact of capsule endoscopy findings in the management of Crohn's Disease. Dig Dis Sci. 2010;55:411-4. https:// doi.org/10.1007/s10620-009-0758-8.

6. Enns RA, Hookey L, Armstrong D et al. Clinical practice guidelines for the use of video capsule endoscopy. Gastroenterology. 2017;152:497-514. https://doi.org/10.1053/j.gastro.2016.12. 032.

7. Lazarev M, Huang C, Bitton A et al. Relationship between proximal Crohn's disease location and disease behavior and surgery: A cross-sectional study of the IBD Genetics Consortium. Am J Gastroenterol. 2013;108:106-12. https://doi.org/10.1038/ajg. 2012.389.

8. Flamant M, Trang C, Maillard O et al. The prevalence and outcome of jejunal lesions visualized by small bowel capsule endoscopy in Crohn's disease. Inflamm Bowel Dis. 2013;19:1390-6. https://doi.org/10.1097/MIB.0b013e3182 $8133 \mathrm{c} 1$.

9. Ben-Horin S, Lahat A, Lahat M M, et al. P392 Comprehensive video capsule endoscopy-based monitoring predicts short and long-term risk of disease flares in small bowel 
Crohn's disease: A prospective cohort study. J Crohn's Colitis. 2018;12:S302-S303.

10. Macedo Silva V, Freitas M, Boal Carvalho et al. APEX score: Predicting flares in small-bowel Crohn's disease after mucosal healing. Dig Dis Sci. (Epub ahead of print). https://doi.org/10. 1007/s10620-021-07148-2.
Publisher's Note Springer Nature remains neutral with regard to jurisdictional claims in published maps and institutional affiliations. 